\title{
Erratum
}

\section{An Apparent Phenomenal Descriptive Method for Judging the Synchronization of Rotation of Binary Stars}

Li Lin-sen

(J. Astrophys. Astr., (2004) Vol. 25, Nos. 3 \& 4, pp. 203-211)

Table 1. The calculated results for synchronous parameters of twenty components in ten eclipsing binary systems by using the apparent descriptive method of formula (4).

\begin{tabular}{lccccccccc}
\hline \multirow{2}{*}{ Name } & & $\begin{array}{c}\text { Sp } \\
\text { type }\end{array}$ & $P(\mathrm{~d})$ & $i(\mathrm{deg})$ & $R_{1,2}\left(R_{\odot}\right)$ & $\begin{array}{c}\left(V_{1,2} \sin i\right)_{M} \\
(\mathrm{~km} / \mathrm{s})\end{array}$ & $Q_{e, e^{\prime}}$ & $P_{R} \gtrless P$ & Synch \\
\hline GT Cep & 1 & B2V & 4.9087 & 78 & 4.64 & 42 & 1.11 & $P_{R}>P$ & $\mathrm{~B}$ \\
& 2 & A0 & & & 6.04 & 58 & 1.05 & $P_{R}>P$ & $\mathrm{~A}$ \\
My Cyg & 1 & Am & 4.0051 & 89 & 2.32 & 26 & 1.13 & $P_{R}>P$ & $\mathrm{~B}$ \\
& 2 & Am & & & 2.06 & 47 & 0.55 & $P_{R}<P$ & $\mathrm{D}$ \\
V451 Oph & 1 & B9V & 2.1966 & 87 & 2.48 & 48 & 1.19 & $P_{R}>P$ & $\mathrm{~B}$ \\
& 2 & A0 & & & 1.98 & 45 & 1.01 & $P_{R}>P$ & $\mathrm{~A}$ \\
U Oph & 1 & B4.5 & 1.6773 & 86.6 & 3.27 & 110 & 0.89 & $P_{R}<P$ & $\mathrm{~B}$ \\
& 2 & B5.5 & & & 3.02 & 100 & 0.91 & $P_{R}<P$ & $\mathrm{~B}$ \\
V505 Sgr & 1 & A2v & 1.8287 & 75 & 2.30 & 100 & 0.95 & $P_{R}<P$ & $\mathrm{~A}$ \\
& 2 & F81V & & & 2.35 & 50 & 1.94 & $P_{R}>P$ & $\mathrm{E}$ \\
CV Vel & 1 & B2V & 6.8925 & 88.2 & 4.27 & 50 & 0.63 & $P_{R}<P$ & $\mathrm{D}$ \\
& 2 & B2V & & & 4.16 & 85 & 0.36 & $P_{R}<P$ & $\mathrm{D}$ \\
CD Tau & 1 & F7V & 3.4351 & 88 & 1.49 & 34 & 0.64 & $P_{R}<P$ & $\mathrm{D}$ \\
& 2 & F7V & & & 1.50 & 34 & 0.65 & $P_{R}<P$ & $\mathrm{D}$ \\
V1143 Cyg & 1 & F5V & 7.6407 & 87 & 1.34 & 9 & 0.98 & $P_{R}<P$ & $\mathrm{~A}$ \\
& 2 & F5V & & & 1.04 & 20 & 0.34 & $P_{R}<P$ & $\mathrm{D}$ \\
ZZ Boo & 1 & F2V & 4.9917 & 88 & 1.76 & 12 & 1.48 & $P_{R}>P$ & $\mathrm{E}$ \\
& 2 & F2V & & & 1.70 & 25 & 0.69 & $P_{R}<P$ & $\mathrm{D}$ \\
AS Cam & 1 & B8V & 3.4309 & 89 & 2.06 & 40 & 0.7 & $P_{R}<P$ & $\mathrm{C}$ \\
& 2 & A0V & & & 2.47 & 30 & 1.21 & $P_{R}>P$ & $\mathrm{C}$ \\
\hline
\end{tabular}




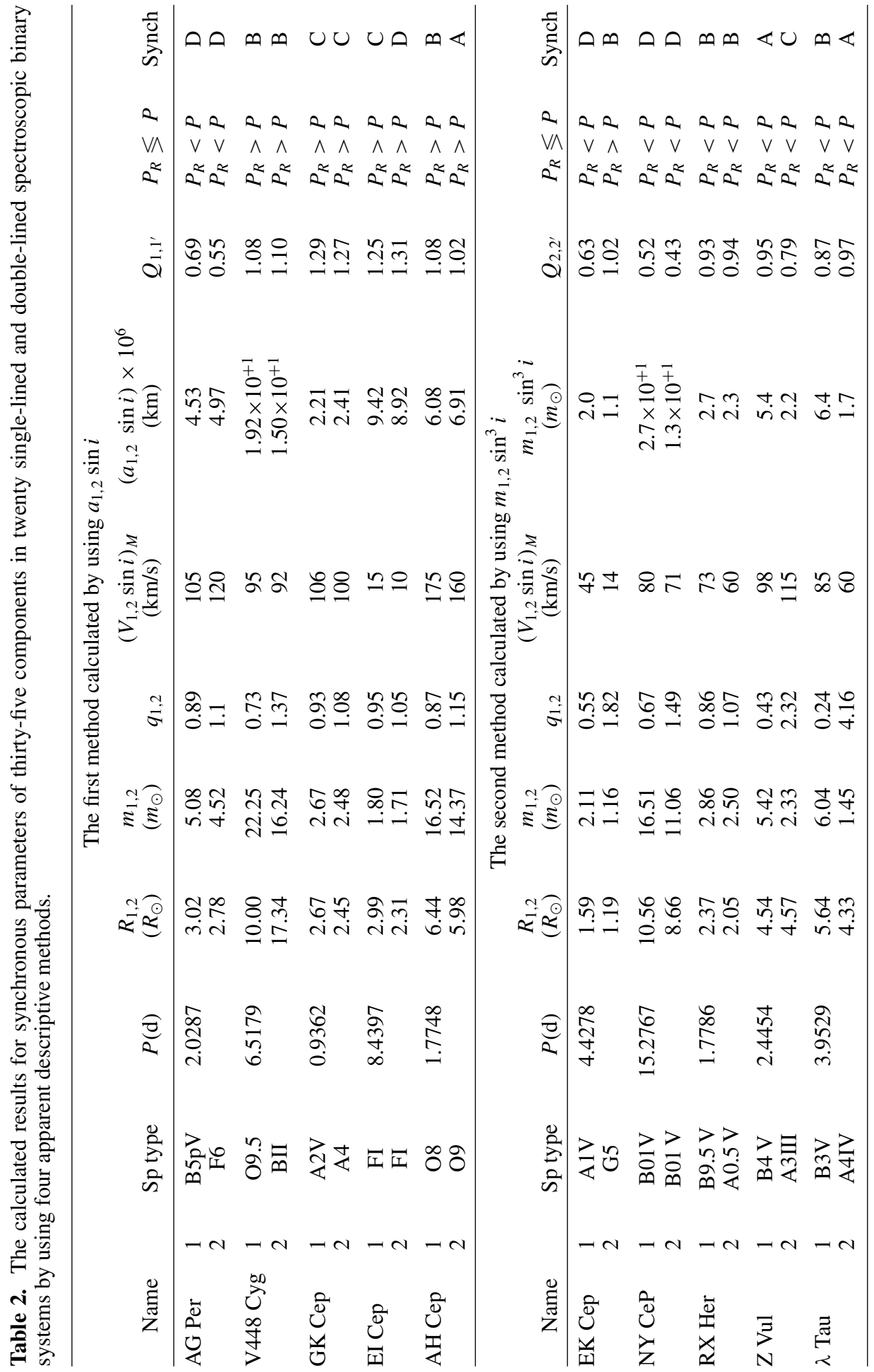




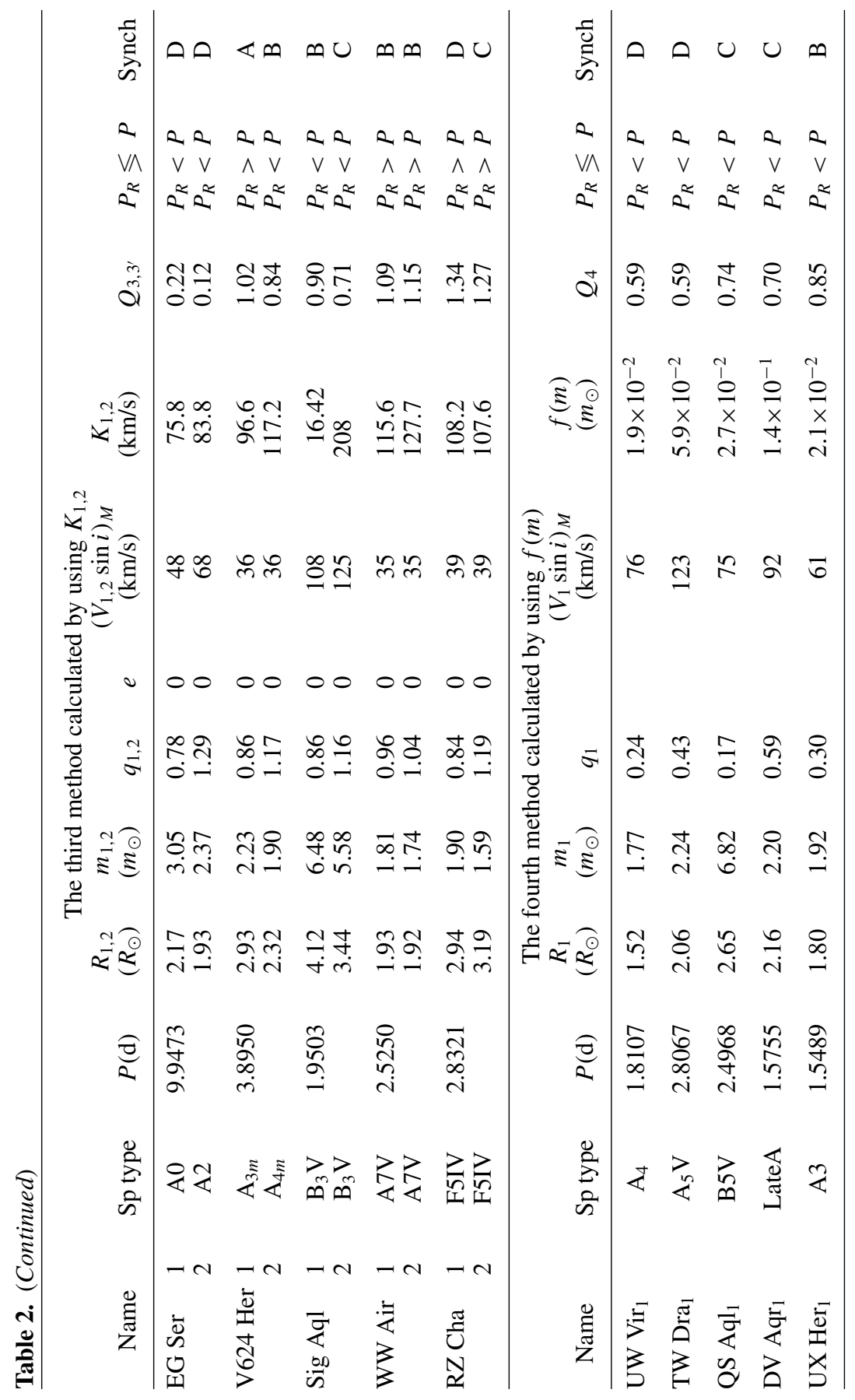


450

\section{References}

Li, Lin-sen, Acta Astrophys. Sinica, 1998, 18, 77; 1997, 17, 407. 